\title{
Review of: "Current practices of management of maternal and congenital Cytomegalovirus infection during pregnancy after a maternal primary infection occurring in first trimester of pregnancy: Systematic review"
}

\author{
ANTONELLA GIANCOTTI ${ }^{1}$
}

1 Sapienza University of Rome

Potential competing interests: The author(s) declared that no potential competing interests exist.

This systematic review aims to provide the state of the art on practices concerning management of maternal and congenital CMV infection during pregnancy, after maternal primary infection (PI) in first trimester of pregnancy.

The article is well written and illustrates management of maternal and congenital CMV during pregnancy in absence of international consensus. The subject is of interest the analysis well performed so i would like to congratulate with authors for their effort. 